\title{
Knee medial and lateral contact forces computed along subject-specific contact point trajectories of healthy volunteers and osteoarthritic patients
}

\author{
Raphael Dumas ${ }^{1[0000-0002-0368-8248]}$, Ali Zeighami ${ }^{2,3}$, Rachid Aissaoui ${ }^{2,3}$ \\ ${ }^{1}$ Univ Lyon, Université Claude Bernard Lyon 1, IFSTTAR, LBMC UMR_T9406, Lyon, \\ France \\ ${ }^{2}$ Laboratoire de Recherche en Imagerie et Orthopédie, Centre de Recherche du CHUM \\ ${ }^{3}$ Département de génie des systèmes, École de Technologie Supérieure, Montréal, Canada \\ raphael.dumas@ifsttar.fr
}

\begin{abstract}
Tibiofemoral medial and lateral contact forces, analysed on healthy volunteers, seem to depend on the trajectories of the contact points. Contact point trajectories in OA patients are shifted in the medial direction and contact forces are generally reported to be slightly reduced.

The present study compares medial and lateral contact forces between OA patients and healthy volunteers. The forces are estimated during gait using a musculoskeletal model with subject-specific contact point trajectories obtained from biplane X-ray images at different flexion angles. Large inter-subject variability was found in the contact point trajectories and the contact forces. Significant but weak correlations were found between the positions of the contact points in the medial-lateral direction and the peaks of medial contact forces for both healthy volunteers and OA patients: the more medial the contact points the lower the forces.

In the literature, when computing the contact forces considering a frontal equilibrium only, the correlation is obviously strong. Relationship between the positions of the contact points and the contact forces is more controversial in studies using deformable knee models. The interactions between altered contact points and contact forces should be further investigated with subject-specific musculoskeletal models.
\end{abstract}

Keywords: Gait analysis musculoskeletal modelling, tibiofemoral loading.

\section{Introduction}

Tibiofemoral medial and lateral contact forces estimated from musculoskeletal modelling depend on the trajectories of the contact points [1-3].

The partition of the total contact forces is generally based on the adduction-adduction moment, the resultant of the musculo-tendon forces spanning the joint, and the position of the contact points in the frontal plane of the tibia. For a given adductionadduction moment (i.e. obtained by inverse dynamics), and a given resultant of the musculo-tendon forces (i.e. obtained by static optimisation or any alternative method), 
the medial and lateral contact forces are then fully determined by the position of the contact points. Moving the contact points in the medial-lateral direction results in altering the contact forces by a gradient up to $0.06 \mathrm{BW} / \mathrm{mm}$ and may also result in unloading one compartment $[1,2]$. When considering this frontal plane equilibrium, the tibiofemoral alignment can be also taken into account. Both contact point positions and alignment can be informed through radiographic analysis [1]. Considering a tri-dimensional (3D) equilibrium of the tibiofemoral joint (i.e. with flexion-extension, adduction-abduction, and internal-external rotation moments) and computing the musculo-tendon forces and contact forces simultaneously somewhat mitigate the dependence of the medial and lateral contact forces on the position of the contact points [3]. This was reported for subject-specific contact point trajectories of healthy volunteers which remain aligned with the medial and lateral plateau centrelines. In the pathologic case such as osteoarthritis (OA), the contact point trajectories appear to be shifted in the medial direction [4] and to demonstrate a lower excursion in the lateral compartment [5].

In the same time, the medial and lateral contact forces have been reported to be generally similar or slightly reduced in OA patients compared to healthy volunteers [6-9]. Only Meireles et al. (2017) reported slightly higher forces in OA patients [10]. In these studies, the contact point trajectories were not subject-specific, either set at two fixed positions in the frontal plane $[6,7]$ or computed by a generic deformable model of the knee [8-10]. Interestingly, Meireles et al. (2017) found that the medial contact point estimated by the deformable knee model at the timing of the first peak had a more posterior-lateral location in severe OA while Van Rossom et al. (2018) reported that the contact locations were not significantly different between OA patients and healthy volunteers.

The objective of the present study is to compare medial and lateral contact forces between OA patients and healthy volunteers using a lower limb musculoskeletal model which include subject-specific contact point trajectories to better understand how they differ and how they can be differently affected by the contact point positions.

\section{$2 \quad$ Material and Methods}

This study includes the data of 10 healthy volunteers $(6 \mathrm{men}, 4$ women, $55 \mathrm{yrs} ., 1.68 \mathrm{~m}$, $71 \mathrm{~kg}$ ) and 12 severe OA patients ( 2 men, 10 women, 59 yrs. $1.61 \mathrm{~m}, 85.53 \mathrm{~kg}$, K-L grade 4). All subjects signed an informed consent form and the experimental protocol was approved by the ethics committees of the Centre de Recherche, Centre Hospitalier de l'Université de Montréal (CRCHUM) and Ecole de Technologie Supérieure de Montréal (ETS). All the research and methods in this study were performed in accordance with the CRCHUM and ETS ethics committee guidelines as well as with the Helsinki Declaration of 1975, as revised in 2000.

The 3D models of their tibia and femur were reconstructed and registered using $\mathrm{EOS}^{\mathrm{TM}}$ low-dose biplane X-ray images at $0^{\circ}, 15^{\circ}, 30^{\circ}, 45^{\circ}$, and $70^{\circ}$ of knee flexion.

A lower limb musculoskeletal model with five joint degrees of freedom and 43 muscle lines of action [11] was scaled to the subject anthropometry using the segment lengths. The subject-specific contact point trajectories were approximated using a 
weighted centre of the bone-to-bone distances [4] and were expressed as a function of the knee flexion angle. These subject-specific contact point trajectories were introduced as kinematic constraints into the musculoskeletal model [3]. The corresponding Lagrange multipliers were the medial and lateral contact forces computed simultaneously with the musculo-tendon forces.

All forces were estimate during $45 \mathrm{~s}$ of gait at comfortable speed using the data recorded by an instrumented split-belt treadmill (AMTI) and reflective markers placed on the body segments and on the kneeKG $\mathrm{G}^{\mathrm{TM}}$ system tracked by a 12-camera Vicon system. The contact forces were normalized to body weight (BW) and to $100 \%$ of gait stance.

Linear regressions were computed between the peaks of medial and lateral contact forces and the positions of the contact points in the medial-lateral direction at the corresponding timings.

\section{$3 \quad$ Results}

\subsection{Contact point trajectories and contact forces}

Figure 1 displays the individual contact point trajectories during weight-bearing quasistatic squat (plotted over the generic bone of the tibia used for the 3D reconstruction [4]) and the individual medial and lateral contact forces during gait for all healthy volunteers and $\mathrm{OA}$ patients.

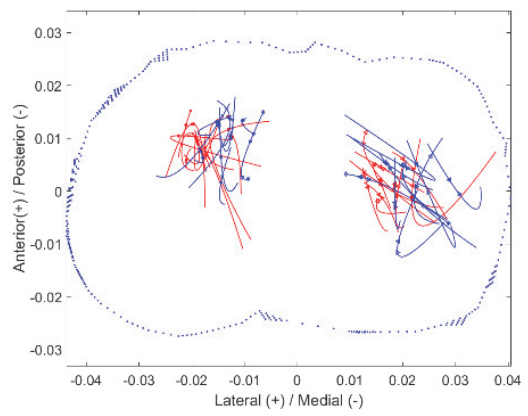

(a)

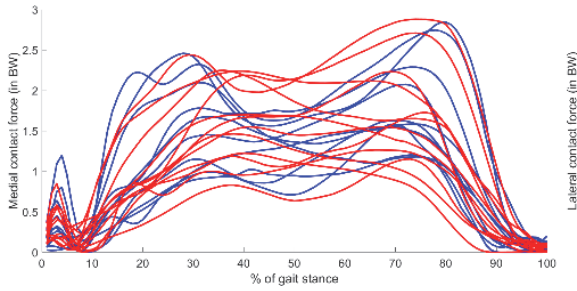

(b)

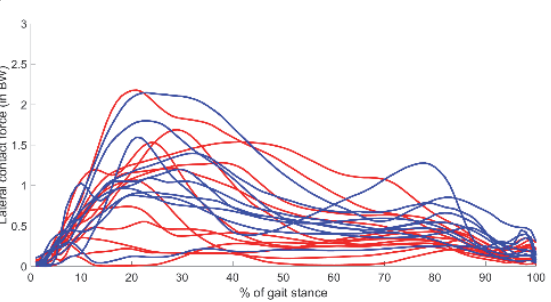

(c)

Fig. 1. (a) Contact point trajectories (in m), (b) medial contact force (in BW), (c) lateral contact force (in BW) for healthy volunteers (in blue) and OA patients (in red). 
The contact point trajectories demonstrated a large inter-subject variability. In the medial compartment, on the medial-lateral axis, the trajectories fell between -6.4 and -26.8 $\mathrm{mm}$ with respect to the tibia centerline for the healthy volunteers and between -5.3 and $-24.8 \mathrm{~mm}$ for the OA patients. In the lateral compartment, the trajectories fell between 9.1 and $34.8 \mathrm{~mm}$ with respect to the tibia centerline for the healthy volunteers and between 10.2 and $37.6 \mathrm{~mm}$ for the OA patients.

The contact forces also demonstrated some inter-subject variability. The medial contact force at first peak was $1.6 \pm 0.5 \mathrm{BW}$ (mean and standard deviation) for the healthy volunteers and $1.6 \pm 0.5 \mathrm{BW}$ for the OA patients. At second peak, the medial contact force was $1.9 \pm 0.6 \mathrm{BW}$ and $1.7 \pm 0.6 \mathrm{BW}$, respectively. The lateral contact force was $1.0 \pm 0.5 \mathrm{BW}$ for the healthy volunteers and $1.0 \pm 0.6 \mathrm{BW}$ for the OA patients at first peak, $0.6 \pm 0.3$ and $0.5 \pm 0.2 \mathrm{BW}$, respectively, at second peak.

\subsection{Linear regressions}

Figure 2 displays the linear regressions obtained between the first and second peaks of medial and lateral contact forces and the positions of the contact points in the mediallateral direction at the corresponding timings. The slopes obtained in the literature by sensitivity analysis on the positions of the contact points (between $+/-16 \mathrm{~mm}$ and $+/$ $24 \mathrm{~mm}[1]$ and between $+/-15 \mathrm{~mm}$ and $+/-35 \mathrm{~mm}$ [2]) are also summarized.

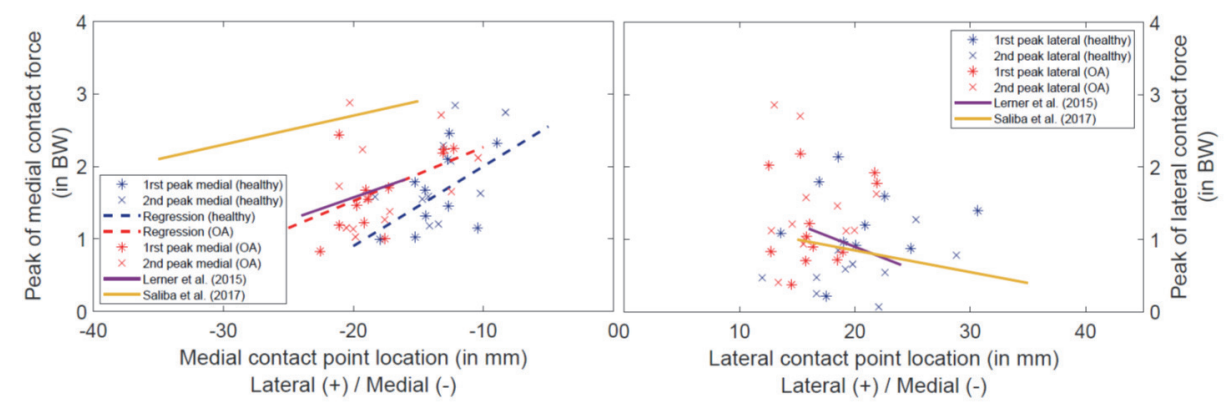

Fig. 2. Regression between first and second peaks of medial and lateral contact forces (in BW) and positions of the contact points in the medial-lateral direction (in $\mathrm{mm}$ ) at the corresponding timings.

Linear regressions were found for the relationship between the positions of the contact points in the medial compartment and the first and second peaks of medial contact forces for both healthy volunteers $\left(R^{2}=0.24, p=0.001\right)$ and OA patients $\left(R^{2}=0.13, p\right.$ $=0.01$ ). The slopes were $0.13 \mathrm{BW} / \mathrm{mm}$ and $0.07 \mathrm{BW} / \mathrm{mm}$, respectively. No linear regression was found for the relationship between the positions of the contact points in the lateral compartment and the peaks of lateral contact forces for both healthy volunteers and OA patients. 


\section{Discussion}

The present study compares medial and lateral contact forces between healthy volunteers and OA patients when introducing subject-specific contact point trajectories in the musculoskeletal model. The mean estimated contact forces were comparable to the forces reported in the literature for similar case-control studies [6-10] and demonstrated slightly lower forces for the OA patient (Table 1).

Table 1. Medial and lateral contact forces reported in the case-control studies of the literature

\begin{tabular}{|c|c|c|c|c|c|c|}
\hline \multirow[b]{2}{*}{ Authors } & & \multirow[b]{2}{*}{$\mathrm{n}$} & \multicolumn{2}{|c|}{ Medial } & \multicolumn{2}{|c|}{ Lateral } \\
\hline & & & $1^{\text {rst }}$ peak & $2^{\text {nd }}$ peak & $1^{\text {rst }}$ peak & $2^{\text {nd }}$ peak \\
\hline \multirow{2}{*}{$\begin{array}{l}\text { Kumar et al. } \\
\text { (2013) }\end{array}$} & Healthy & 16 & 2.4 & 1.8 & 1.3 & 0.5 \\
\hline & $\mathrm{OA}$ & 12 & 2.6 & 2.1 & 0.9 & 0.1 \\
\hline \multirow{2}{*}{$\begin{array}{l}\text { Sritharan et al. } \\
\text { (2017) }\end{array}$} & Healthy & 19 & 2.0 & 3.0 & 0.3 & 0.5 \\
\hline & $\mathrm{OA}$ & 39 & 2.1 & 3.2 & 0.3 & 0.6 \\
\hline \multirow{2}{*}{$\begin{array}{l}\text { Dell'Isola et al. } \\
\text { (2017) }\end{array}$} & Healthy & 18 & \multicolumn{2}{|c|}{2.1} & \multicolumn{2}{|c|}{1.4} \\
\hline & $\mathrm{OA}$ & 17 & \multicolumn{2}{|c|}{2.0} & \multicolumn{2}{|c|}{1.1} \\
\hline \multirow{3}{*}{$\begin{array}{l}\text { Meireles et al. } \\
(2017)\end{array}$} & Healthy & 17 & 2.2 & 1.8 & 1.2 & 1.2 \\
\hline & Early OA & 14 & 2.6 & 1.9 & 1.4 & 1.5 \\
\hline & $\mathrm{OA}$ & 16 & 2.8 & 2.1 & 1.3 & 1.6 \\
\hline \multirow{3}{*}{$\begin{array}{l}\text { Van Rossom } \\
\text { et al. (2018) }\end{array}$} & Healthy & 19 & 1.8 & 1.9 & 1.3 & 1.0 \\
\hline & OA (Medial) & 8 & 1.9 & 1.8 & 1.1 & 0.8 \\
\hline & OA (Lateral) & 7 & 1.5 & 1.6 & 1.2 & 1.0 \\
\hline \multirow{2}{*}{ Present study } & Healthy & 10 & 1.6 & 1.9 & 1.0 & 0.6 \\
\hline & $\mathrm{OA}$ & 12 & 1.6 & 1.7 & 1.0 & 0.5 \\
\hline
\end{tabular}

For both healthy volunteers and OA patients, a large inter-subject variability was found in the contact point trajectories and the contact forces but without a strong covariation. This may be due to the fact that the contact point trajectories were measured during weight-bearing quasi-static squat while the contact forces are estimated during gait. Yet, a recent systematic review showed that the contact point trajectories obtained by biplane X-ray images during quasi-static squat matched the contact point trajectories obtained by fluoroscopy, MRI or CT during other activities (gait, step-up, kneeling, lunge) [5]. It is possible, in both healthy volunteers and OA patients, to measure the contact point trajectories during gait using bi-plane fluoroscopy [12], however, to the authors' knowledge, this information has never been introduced in subject-specific musculoskeletal model so far.

When computing the contact forces considering a frontal equilibrium only, the contact forces are directly determined by the contact point positions (proportional to the inverse of the contact point positions). Conversely, in the present study, weak correlations were found in the medial compartment and no correlation at all in the lateral compartment. Still, the slopes obtained by sensitivity analysis [1, 2] and the one obtained in the present study are comparable, although higher for the healthy volunteers. The fact Meireles et al. (2017) found, in the medial compartment for OA patients, higher 
contact forces and, in the same time, more lateral contact points is also consistent in terms of variation.

The weak correlation and lower slope for OA demonstrate that the contact forces are potentially governed by other parameters such as joint moments and knee alignment as reported in the literature [13-15]. Because the contact point trajectories are introduced as kinematic constraints into the lower limb musculoskeletal model, not only the position of the contact points but also the joint kinematics (i.e. including the adductionadduction angle) is subject-specific. The relationship between knee alignment and the contact forces is therefore not expected to be strong for the OA patients of the present study but this should be tested in further analyses. Another sensitivity analysis in the literature [16] also reported a relationship between the position of the contact points in the anterior-posterior direction and the contact forces and, again, this should be tested in further analyses.

This study has some limitations. The study sample is small. It ranges between 12 and 39 (Table 1) in the previous studies comparing medial and lateral contact forces between healthy volunteers and OA patients [6-9]. Only the contact point trajectories were subject-specific. The musculoskeletal geometry was only scaled. However, it is important to keep in mind that the contact point trajectories were introduced as kinematic constraints leading also to subject-specific tibiofemoral kinematics. These contact point trajectories (and the kinematics) were measured during weight-bearing quasistatic squat and not during walking but it can be expected that the same inter-subject variability (especially for the OA patients) could be observed during gait.

\section{Conclusion}

In the present study, like in most case-control studies of the literature, medial and lateral contact forces during gait appears to be slightly lower in OA patients compared to healthy volunteers. Based on the subject-specific contact point trajectories, weak correlations were found between the positions of the contact points and the peaks of contact forces for both healthy volunteers and OA patients: the more medial the contact points the lower the forces in the medial compartment.

In the literature, when computing the contact forces considering a frontal equilibrium only, the correlation is obviously strong. Relationship between the positions of the contact points and the contact forces is more controversial in studies using deformable knee models. The interactions between altered contact points and contact forces should be further investigated with subject-specific musculoskeletal models.

\section{Acknowledgments}

Fonds de Recherche du Quebec en Sante (FRQ-S), Fonds de Recherche du Quebec en Nature et Technologie (FRQNT), Natural Science and Research Council of Canada (NSERC), LABEX PRIMES (ANR-11-LABX-0063) of Université de Lyon. 


\section{References}

1. Lerner, Z.F., DeMers, M.S., Delp, S.L., Browning, R.C.: How tibiofemoral alignment and contact locations affect predictions of medial and lateral tibiofemoral contact forces. Journal of Biomechanics 48, 644-650 (2015)

2. Saliba, C.M., Brandon, S.C.E., Deluzio, K.J.: Sensitivity of medial and lateral knee contact force predictions to frontal plane alignment and contact locations. Journal of Biomechanics 57 , 125-130 (2017)

3. Zeighami, A., Aissaoui, R., Dumas, R.: Knee medial and lateral contact forces in a musculoskeletal model with subject-specific contact point trajectories. Journal of Biomechanics 69, 138-145 (2018)

4. Zeighami, A., Dumas, R., Kanhonou, M., Hagemeister, N., Lavoie, F., de Guise, J.A., Aissaoui, R.: Tibio-femoral joint contact in healthy and osteoarthritic knees during quasi-static squat: A bi-planar X-ray analysis. Journal of Biomechanics 53, 178-184 (2017)

5. Scarvell, J.M., Galvin, C.R., Perriman, D.M., Lynch, J.T., van Deursen, R.W.M.: Kinematics of knees with osteoarthritis show reduced lateral femoral roll-back and maintain an adducted position. A systematic review of research using medical imaging. Journal of Biomechanics 75, 108-122 (2018)

6. Dell'Isola, A., Smith, S.L., Andersen, M.S., Steultjens, M.: Knee internal contact force in a varus malaligned phenotype in knee osteoarthritis (KOA). Osteoarthritis and Cartilage 25, 2007 $2013(2017)$

7. Kumar, D., Manal, K.T., Rudolph, K.S.: Knee joint loading during gait in healthy controls and individuals with knee osteoarthritis. Osteoarthritis and Cartilage 21, 298-305 (2013)

8. Sritharan, P., Lin, Y.-C., Richardson, S.E., Crossley, K.M., Birmingham, T.B., Pandy, M.G.: Musculoskeletal loading in the symptomatic and asymptomatic knees of middle-aged osteoarthritis patients. Journal of Orthopaedic Research 35, 321-330 (2017)

9. Van Rossom, S., Khatib, N., Holt, C., Van Assche, D., Jonkers, I.: Subjects with medial and lateral tibiofemoral articular cartilage defects do not alter compartmental loading during walking. Clinical Biomechanics 60, 149-156 (2018)

10.Meireles, S., Wesseling, M., Smith, C.R., Thelen, D.G., Verschueren, S., Jonkers, I.: Medial knee loading is altered in subjects with early osteoarthritis during gait but not during step-upand-over task. PLOS ONE 12, e0187583 (2017)

11.Moissenet, F., Chèze, L., Dumas, R.: A 3D lower limb musculoskeletal model for simultaneous estimation of musculo-tendon, joint contact, ligament and bone forces during gait. Journal of Biomechanics 47, 50-58 (2014)

12.Farrokhi, S., Voycheck, C.A., Gustafson, J.A., Fitzgerald, G.K., Tashman, S.: Knee joint contact mechanics during downhill gait and its relationship with varus/valgus motion and muscle strength in patients with knee osteoarthritis. The Knee 23, 49-56 (2016)

13.Manal, K., Gardinier, E., Buchanan, T.S., Snyder-Mackler, L.: A more informed evaluation of medial compartment loading: the combined use of the knee adduction and flexor moments. Osteoarthritis and Cartilage 23, 1107-1111 (2015)

14.Richards, R.E., Andersen, M.S., Harlaar, J., van den Noort, J.C.: Relationship between knee joint contact forces and external knee joint moments in patients with medial knee osteoarthritis: effects of gait modifications. Osteoarthritis and Cartilage 26, 1203-1214 (2018) 
15.Trepczynski, A., Kutzner, I., Bergmann, G., Taylor, W.R., Heller, M.O.: Modulation of the Relationship Between External Knee Adduction Moments and Medial Joint Contact Forces Across Subjects and Activities. Arthritis \& Rheumatology 66, 1218-1227 (2014)

16.Nissan, M.: Review of some basic assumptions in knee biomechanics. Journal of Biomechanics 13, 375-381 (1980) 